\title{
Measuring the Voltage Dependence of Current Transformers
}

This paper was downloaded from TechRxiv (https://www.techrxiv.org).

\section{LICENSE}

CC BY 4.0

SUBMISSION DATE / POSTED DATE

$21-12-2020 / 30-12-2020$

\section{CITATION}

van den Brom, Helko; van Leeuwen, Ronald; Rietveld, Gert; Hoogenboom, Dennis (2020): Measuring the Voltage Dependence of Current Transformers. TechRxiv. Preprint.

https://doi.org/10.36227/techrxiv.13469673.v1

$\mathrm{DOI}$

10.36227/techrxiv.13469673.v1 


\title{
Measuring the Voltage Dependence of Current Transformers
}

\author{
Ronald van Leeuwen, Helko van den Brom, Gert Rietveld, Ernest Houtzager and Dennis Hoogenboom \\ VSL, Delft, The Netherlands \\ hvdbrom@vsl.nl
}

\begin{abstract}
A setup has been developed to determine the voltage dependence of the ratio error and phase displacement of current transformers (CTs) used for medium- or high-voltage (HV) applications. A shielded $\mathrm{HV}$ cable is used to protect the reference CT for the voltage applied to the CT under test. As a first step, a method was developed to determine and correct for the effect of the leakage current through this cable on the ratio error and phase displacement of the reference CT for voltages up to $24 \mathrm{kV}$.
\end{abstract}

Index Terms - Current measurement, current transformers, measurement standards, precision measurements.

\section{INTRODUCTION}

The accuracy and reliability of current transformers (CTs) is very important when measuring the energy use for billing purposes, e.g., in railway supply systems, or for monitoring grid stability in electricity distribution grids. In these applications, CTs are typically connected with their input terminals at high potential. However, their ratio error and phase displacement are usually calibrated with all terminals at ground potential, thus neglecting the influence of leakage currents.

A current-comparator-based setup has been developed in the past for calibrating CTs under actual high-voltage (HV) operating conditions [1], but this system is rather complicated and difficult to establish. Furthermore, it is not unambiguously proven that the reference system itself has negligible voltage dependence. In this paper an alternative, metrologically sound, method is presented to determine the voltage dependence of CTs. As a first step of the development, the effect of the high potential on the reference CT is determined.

\section{MEASUREMENT SETUP}

\section{A. Measurement setup for CT calibration at high potential}

The measurement setup investigated in this paper is a modified version of a setup developed for the calibration of the complex current ratio $n$ (reflecting the ratio and phase displacement) of high-current CTs at ground potential [2]. The operating principle of the original setup is to compare the complex ratio errors $\varepsilon=n \cdot I_{s} / I_{p}-1$ of a CT under test and a reference CT by driving the same current $I_{p}$ through their primary windings in series and to measure the ratio of the two secondary currents $I_{s}$. The secondary current ratio is determined using a step-down transformer and a current buffer converting these currents to voltage.

The reference CT used in the setup is a three-stage electronically compensated CT [3] with a ratio error less than two parts in $10^{6}$ in magnitude and $2 \mu \mathrm{rad}$ in phase at $50 \mathrm{~Hz}$ for currents up to $2.5 \mathrm{kA}$. The output voltages of the current buffers are measured using two high-precision sampling voltmeters. The ratio of the secondary currents and can be determined with an extended uncertainty of better than two parts in $10^{6}$.

In the modified setup, a potential is applied to the primary winding of the CT under test by means of a high-voltage source that is synchronized with the primary current source (see Fig. 1). A shielded HV cable is used to protect the reference CT for the high voltages applied to the unit under test.

\section{$B$. Voltage dependence of the reference $C T$}

The voltage dependence of the ratio error and phase displacement of a CT is due to leakage currents caused by the potential difference between the primary current circuit and the secondary output circuit. Since there is also a leakage path to ground in the high-voltage cable insulation, we must verify its influence on the accuracy of the reference CT.

To investigate the voltage dependence of the ratio error of the reference CT, we used two identical reference CTs positioned closely together halfway the shielded HV cable. If only one side of the cable shield is grounded, only the leakage current originating from the opposite part of the cable will flow through the primary winding of both reference CTs. Apart from the primary current itself, the reference CTs will therefore sense approximately the same leakage current, which is about half of the total leakage current in this configuration.

To determine the size of the leakage current and its influence on the ratio error of the reference $\mathrm{CT}$, the grounding cable connected to the shield at the end of the cable is fed back through the primary winding of only one of the two reference CTs. Therefore, the two reference CTs will sense a different

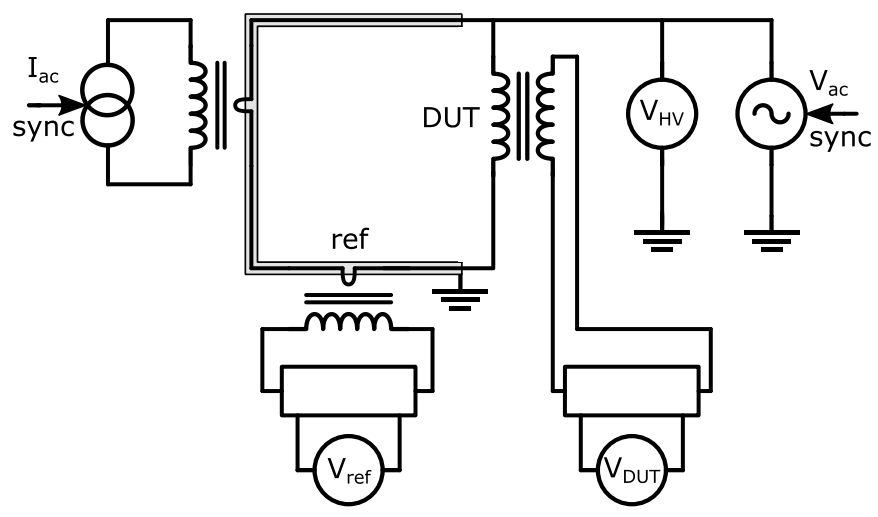

Fig. 1. Simplified schematic overview of the setup to measure the voltage dependence of CTs. A grounded shield prevents the reference CT to sense the high potential applied to the primary current circuit. 
current through their primary winding, the difference being the total leakage current $I_{l e a k}$ through the cable. As a consequence, the observed ratio error $\varepsilon=n \cdot I_{s} /\left(I_{p} \pm 1 / 2 \cdot I_{\text {leak }}\right)-1$ will be different as well, the difference changing with the applied voltage. Hence, when determining the ratio error difference between the two reference CTs while increasing the voltage applied to the primary current circuit, the magnitude and phase of the leakage current can be determined as a function of applied voltage.

\section{PRELIMINARY MEASUREMENT RESULTS}

Preliminary measurements of the difference in ratio error and phase displacement between the two reference CTs have been performed, one of which having the ground cable fed back through the primary winding as described in the last part of section II.B. As a first verification, the complex ratio error difference between the two reference CTs was measured without leading the ground cable through the CTs for a primary current of $100 \mathrm{~A}$ at $53 \mathrm{~Hz}$ with an applied voltage up to $24 \mathrm{kV}$ in phase with the primary current. The voltage dependence of the difference in ratio error and phase displacement between the two CTs was measured to be less than a few parts in $10^{7}$ and $0.1 \mu \mathrm{rad}$, respectively, over the whole voltage range. The same result was obtained when applied voltage and primary current were 90 degrees out of phase.

For the configuration with the grounding cable led through the primary winding of one of the two reference CTs, large differences were observed, however. The first measurements were carried out with the applied voltage in phase with the primary current. The difference in ratio error was still measured to be less than one part in $10^{6}$ over the whole voltage range. The phase displacement difference between the two CTs, however, scaled linearly with voltage, with a linearity constant of $4.2 \mu \mathrm{rad} / \mathrm{kV}$, up to slightly higher than $100 \mu \mathrm{rad}$ at $24 \mathrm{kV}$.

For the same configuration, when setting the phase between primary current and applied voltage to 90 degrees, the ratio error difference scaled linearly with voltage, with a linearity constant of $4.210^{-6} / \mathrm{kV}$, up to a difference of just above 100 parts in $10^{6}$ (corresponding to a leakage current of $10 \mathrm{~mA}$ ) at $24 \mathrm{kV}$. In this case the phase displacement difference was measured to be less than $1 \mu \mathrm{rad}$ over the whole voltage range.

\section{DISCUSSION}

The leakage current is expected to have maximum influence on the reference CT ratio error if it is in phase with the applied primary current injected with the current source. However, if leakage current and applied primary current are 90 degrees out of phase, it contributes to the ratio error as a second order effect. The opposite holds for the observed phase displacement, which is not changed if the leakage current is in phase with the applied voltage, whereas the influence is largest for a 90 degrees phase difference between leakage current and applied current.
Therefore, the obtained measurement results can be explained if the leakage current through the cable is purely capacitive, such that the leakage current is 90 degrees out of phase with the applied voltage. In this case, the ratio error is mainly influenced if the primary current and the applied voltage are out of phase and the phase displacement is mainly influenced if they are in phase, which is what we observed.

The magnitude of the leakage current will then scale linearly with the applied voltage, $I_{\text {leak }}=\omega C \cdot V$, where $\omega$ denotes the angular frequency of the applied current and voltage and $C$ is the capacitance between the inner conductor of the cable and its shield. The linearity constant obtained from the measurements corresponds to a cable capacitance value of $1.28 \mathrm{nF}$, which is within $1 \%$ of the value measured using a precision LCR meter.

\section{CONCLUSION AND FUTURE WORK}

In this paper we presented a setup to determine the voltage dependence of the ratio error and phase displacement of CTs used for medium- or high-voltage applications. The reference $\mathrm{CT}$ senses the primary current through a shielded HV cable with its shield to ground. A method was developed to determine the magnitude and phase of the leakage current through the highvoltage cable. Preliminary measurements show that it is possible to correct for the effect of this leakage current on the ratio error and phase displacement of the reference CT.

More measurements need to be performed to show the current dependence of the results, especially at higher currents where the relative effect of the leakage current is expected to be lower. Furthermore, the new setup needs to be demonstrated when used for measuring the voltage dependence of CTs under test at high potential.

\section{ACKNOWLEDGEMENT}

The research leading to the results described in this paper is performed within the 16ENG04 MyRails project of the European Metrology Programme for Innovation and Research (EMPIR). The EMPIR initiative is co-funded by the European Union's Horizon 2020 research and innovation programme and the EMPIR participating states.

\section{REFERENCES}

[1] E. So, R. Arseneau, D. Bennett, M.E. Frigault, "A currentcomparator-based system for calibrating high-voltage current transformers under actual operating conditions", IEEE Trans. Instrum. Meas., vol. 60, no. 7, pp. 2449 - 2454, July 2001.

[2] H. E. van den Brom, G. Rietveld, and E. So, "Sampling Current Ratio Measurement System for Calibration of Current Sensors up to $10 \mathrm{kA}$ with $5 \cdot 10^{-6}$ Uncertainty", IEEE Trans. Instrum. Meas, vol. 64, no. 6, pp. 1685-1691, June 2015.

[3] E. So and D. Bennett, "Compact wideband high-current ( $\geq 1000 \mathrm{~A}$ ) multistage current transformers for precise measurements of current harmonics," IEEE Trans. Instrum. Meas., vol. 56, no. 2, pp. 584-587, Apr. 2007. 